\title{
Estado de la sensibilidad a los insecticidas de uso en salud pública en poblaciones naturales de Aedes aegypti (Diptera: Culicidae) del departamento de Casanare, Colombia
}

\author{
Susanne Ardila-Roldán ${ }^{1}$, Liliana Santacoloma², Helena Brochero ${ }^{1}$ \\ 1 Facultad de Agronomía, Universidad Nacional de Colombia, Bogotá, D.C., Colombia \\ 2 Laboratorio de Entomología, Dirección de Red Nacional de Laboratorios, Instituto Nacional de Salud, Bogotá, \\ D.C., Colombia
}

Introducción. La resistencia fisiológica de Aedes aegypti constituye una de las principales amenazas para la eficacia de los programas de control de la transmisión del dengue.

Objetivos. Determinar el estado de la sensibilidad a los insecticidas en poblaciones naturales de $A$. aegypti de tres localidades endémicas para dengue en Casanare.

Materiales y métodos. Se obtuvieron mosquitos adultos de $A$. aegypti a partir de estados inmaduros recolectados en siete poblaciones naturales correspondientes a tres municipios. La primera generación filial fue empleada para evaluar los mecanismos bioquímicos asociados con alteración de $\beta$-esterasas inespecíficas y enzimas monooxigenasas del grupo citocromo P450. La segunda generación permitió evaluar la sensibilidad a los insecticidas a partir de bioensayos, usando la metodología propuesta por los Centers for Disease Control and Prevention para mosquitos adultos y la técnica para larvas de la Organización Mundial de la Salud.

Resultados. Todas las poblaciones evaluadas registraron resistencia al organoclorado DDT y a los piretroides lambdacialotrina y permetrina. Dos presentaron sensibilidad a la deltametrina y cinco a la ciflutrina. Se registró sensibilidad a los organofosforados temefos, malatión y fenitrotión. Ninguna población incrementó las $\beta$-esterasas, pero sí incrementaron las enzimas P450 en dos poblaciones de Yopal.

Conclusiones. Los resultados sugieren que las enzimas P450 pueden jugar un papel importante en la resistencia a los piretroides y al DDT; aun así, otros mecanismos de resistencia pueden estar actuando en las poblaciones. La sensibilidad a los insecticidas organofosforados permitiría continuar con el uso de este grupo químico para interrumpir la transmisión del dengue en Casanare.

Palabras clave: Aedes aegypti, dengue, insecticidas, resistencia a los insecticidas.

doi: http://dx.doi.org/10.7705/biomedica.v33i3.1534

Status of insecticide susceptibility of public health use in natural populations of Aedes aegypti (Diptera: Culicidae) of Casanare, Colombia

Introduction: Physiological resistance of Aedes aegypti is a major threat to effective control programs in the transmission of dengue virus.

Objective: To determine the status of susceptibility to insecticides used in public health, in natural populations of $A$. aegypti from three endemic dengue localities of Casanare.

Materials and methods: Adult mosquitoes were recovered from $A$. aegypti immature stages from seven natural populations collected for three municipalities. The first filial generation was used to assess the biochemical mechanisms associated with loss of susceptibility: nonspecific esterase (NSE) and enzyme cytochrome P450 monooxygenases group. The second filial generation allowed us to evaluate the susceptibility to insecticides from bioassays using the CDC 1998 methodology for adult mosquitoes and WHO 1981 technique for larvae.

Results: In the seven adult populations recorded loss of susceptibility to organochlorine DDT and pyrethroids lambda-cyhalothrin and permethrin. Two populations showed susceptibility to deltamethrin and five populations showed susceptibility to cyfluthrin. There was a susceptibility to organophosphates temephos, malathion and fenitrothion in all populations. No population showed increased NSE but an increase of P450 in two populations of Yopal.

\section{Contribución de los autores:}

Susanne Ardila formuló el proyecto de investigación, realizó el trabajo de campo y redactó el artículo. Liliana Santacoloma asesoró el establecimiento de las colonias e hizo las pruebas biológicas y bioquímicas. Helena Brochero dirigió el proyecto de investigación y corrigió el artículo. 
Conclusions: It appears that the P450 may play an important role in resistance to pyrethroids and DDT, still other resistance mechanisms may be acting in populations. Susceptibility to organophosphate allows continued use of this chemical group to interrupt transmission of dengue in Casanare.

Keywords: Aedes aegypti, dengue, insecticides, insecticide resistance.

doi: http://dx.doi.org/10.7705/biomedica.v33i3.1534

La transmisión del virus del dengue en América se debe a la picadura del mosquito Aedes (Stegomyia) aegypti (Linneaus, 1762). La incidencia de esta virosis se ha incrementado en el mundo desde la década de 1950 (1), estimándose que se infectan entre 50 y 100 millones de personas con el virus del dengue cada año (2). En Colombia, en el 2010, considerado como un año epidémico, se notificaron 146.354 casos de dengue (321,6 por 100.000 habitantes) y 5.420 de dengue grave (11,9 por 100.000 habitantes), de los cuales, el departamento de Casanare aportó 2.127 casos de dengue (653,2 casos por 100.000 habitantes) y 59 casos de dengue grave $(18,2$ casos por 100.000 habitantes), cifras superiores al promedio nacional (3). En Casanare el dengue es endémico desde la década de 1980, cuando se presentaron los primeros brotes de la enfermedad en la ciudad de Yopal (comunicación personal, Secretaría de Salud del Casanare, 2008), posicionándose entre los primeros departamentos que concentran el 75 $\%$ de los casos del país $(4,5)$.

La propagación de esta enfermedad se ve influenciada por factores sociales, como el crecimiento de las poblaciones humanas, el incremento en los viajes nacionales o internacionales, el transporte transcontinental de mercancías, la introducción de nuevos subtipos del virus, los cambios de hábitos en el uso de agua doméstica, la carencia de políticas en salud pública, y la carencia de fuentes humanas y financieras para implementar medidas de control eficientes (6). Ante la ausencia de una vacuna eficaz y la adaptación del mosquito a los entornos humanos, la Organización Mundial de la Salud (OMS) recomienda enfoques estratégicos enmarcados en la promoción de la salud, y el trabajo intersectorial con entidades gubernamentales y no gubernamentales, $y$ una intensificación de la participación comunitaria que permitan lograr acciones sostenibles en el tiempo.

\section{Correspondencia:}

Susanne Ardila-Roldán, Maestría de Ciencias AgrariasEntomología, Facultad de Agronomía, Universidad Nacional de Colombia, Carrera 30 № 45-03, edificio 500, Bogotá, D.C., Colombia

Teléfono: (311) 2817969

dimidiata@gmail.com y scardilar@unal.edu.co

Recibido: 31/12/12; aceptado:08/05/13
La continua presión química ejercida sobre $A$. aegypti ha generado resistencia en poblaciones de 41 naciones de América y el Caribe (7-10) y de otros lugares del mundo $(11,12)$; además, se estima que esta especie es resistente a 24 compuestos diferentes documentados (12). En Colombia, en estudios anteriores también se ha confirmado la presencia de resistencia en poblaciones de este vector en los departamentos de Antioquia, Atlántico, Caquetá, Cauca, Chocó, Cundinamarca, Guaviare, Huila, Meta, Nariño, Putumayo, Santander y Valle del Cauca, en los cuales se documenta la resistencia al dicloro-difenil-tricloroetano (DDT), piretroides y organofosforados (13-16).

EI DDT fue uno de los primeros químicos en emplearse para el control de vectores como $A$. aegypti. La resistencia generada a este compuesto en Colombia y en otras partes del mundo, fue una de las causas, entre otras, que llevó a la reaparición del dengue en la década de 1960 (17). En Colombia, en estudios previos en poblaciones de los departamentos de Antioquia, Chocó y Putumayo, se señaló que la resistencia a este químico se debe probablemente a mecanismos fisiológicos por incremento del grupo enzimático de la glutatión-S-transferasa (15).

En Casanare, el control de la enfermedad es responsabilidad de las autoridades departamentales de salud y se ha basado en la aplicación de productos químicos sintéticos, implementados desde mediados de la década de 1980. Hasta 1992, se utilizó el organoclorado DDT que, posteriormente, fue reemplazado por piretroides como lambdacialotrina y deltametrina (18). Posteriormente y hasta la fecha, el temefos (granulado $1 \mathrm{mg} / \mathrm{L}$ ) como larvicida y el malatión como adulticida, son los únicos químicos que se han empleado para el control del dengue $(19,20)$.

La vigilancia entomológica se basa en el levantamiento de índices de infestación larvaria según la presencia o ausencia de la especie en criaderos domésticos, por lo que el larvicida temefos también se utiliza para reducir las poblaciones del mosquito.

El departamento registra infestación por $A$. aegypti en 18 de sus 19 municipios y, debido 
a la larga historia de limitaciones de agua para consumo humano por carencia de acueducto, es muy arraigado el hábito de almacenar agua en las viviendas. Se desconoce el estado de la sensibilidad a los insecticidas en poblaciones naturales de $A$. aegypti de Casanare, por lo que se dificulta planificar acciones de rotación, mezcla o inhibición de moléculas insecticidas que permitan la eficacia en la reducción de la transmisión de la enfermedad durante brotes o epidemias.

El objetivo de este estudio fue evaluar el estado de la sensibilidad a los insecticidas de uso en salud pública, en poblaciones naturales de $A$. aegypti recolectadas en los municipios de Yopal, Aguazul y Villanueva, del departamento del Casanare.

\section{Materiales y métodos}

\section{Sitios de estudio}

El departamento de Casanare se encuentra ubicado en la región de la Orinoquia, está ubicado entre los $04^{\circ} 17^{\prime} 25^{\prime \prime}$ y los $06^{\circ} 20^{\prime} 45^{\prime \prime}$ de latitud norte y los $69^{\circ} 50^{\prime} 22^{\prime \prime}$ y $73^{\circ} 04^{\prime} 33$ de longitud oeste (21). Tiene una superficie total de $44.640 \mathrm{~km}^{2}$, una temperatura media anual que oscila entre 23 y $29{ }^{\circ} \mathrm{C}$, y un régimen de lluvias monomodal con los meses de mayor precipitación entre marzo y noviembre (humedad relativa: 80-96 \%) (22).

El estudio se llevó a cabo en los municipios de Yopal, Aguazul y Villanueva, seleccionados con los siguientes criterios de inclusión: índices entomológicos de Breteau mayores de 5, altas tasas de incidencia para dengue en el departamento y frecuente aplicación de insecticidas químicos para el control del dengue.

Con estos mismos criterios, en cada municipio se seleccionaron localidades para la recolección de formas inmaduras del mosquito. Cada localidad agrupó diferentes barrios que correspondieron a una unidad geográfica para la vigilancia epidemiológica yentomológica, denominadas "comunasosectores". Una comuna o sector correspondió a un grupo de viviendas que concentraba varios barrios y que compartían características sociales, económicas 0 culturales similares que las diferenciaban de otras agrupaciones.

En este contexto, dentro de un mismo municipio se evaluaron varios sectores o comunas que representaron diferentes poblaciones de $A$. aegypti (figura 1). Se asumió una distancia mínima de $1 \mathrm{~km}$

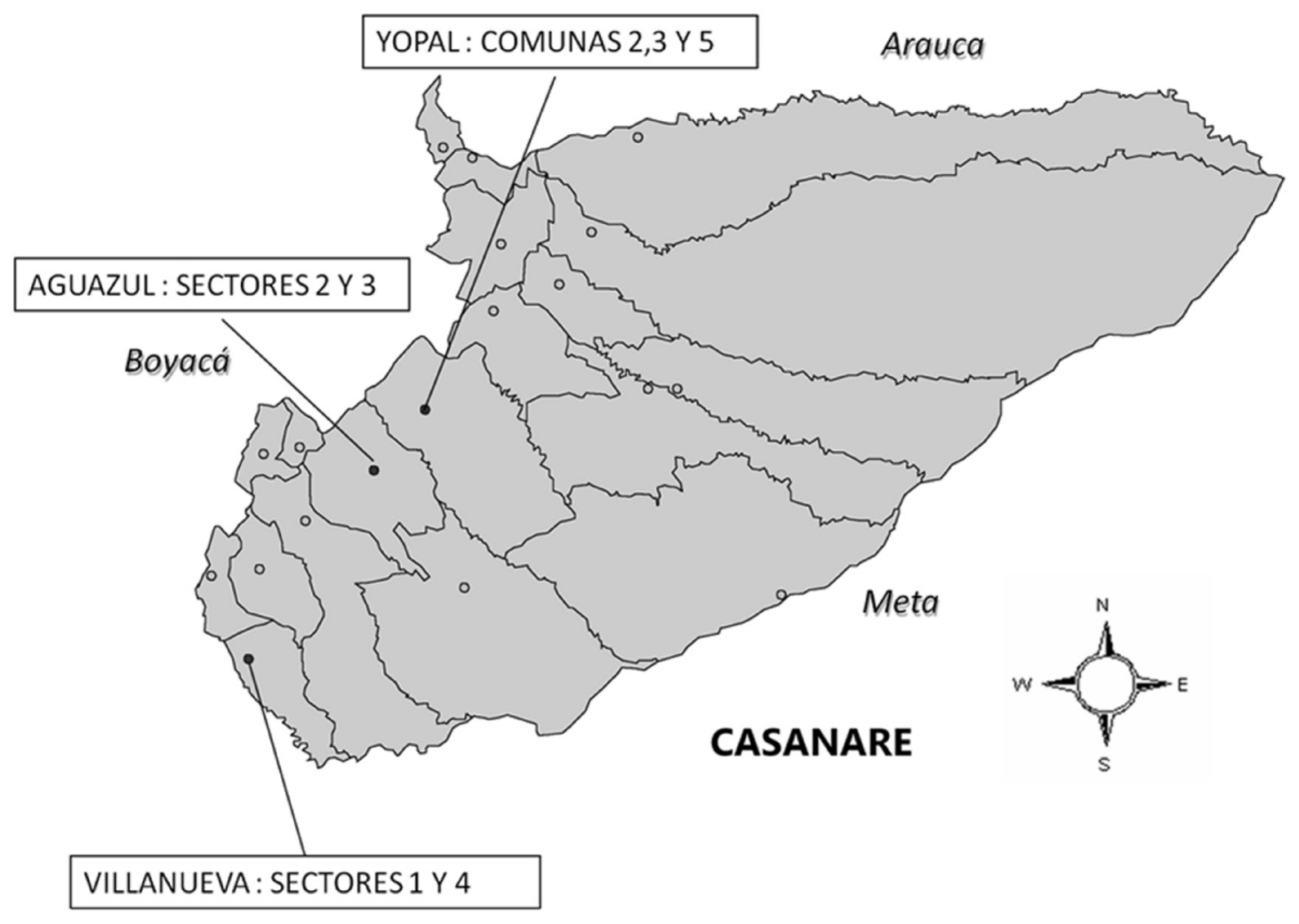

Figura 1. Localidades evaluadas en el departamento de Casanare 
entre cada sitio de recolección de formas inmaduras del insecto, con el propósito de diferenciar genéticamente una población de otra, teniendo en cuenta que el rango de vuelo de la hembra de esta especie rara vez llega a más de 50 a 100 metros de la vivienda humana $(23,24)$.

\section{Recolección de material entomológico}

En cada localidad se hizo un muestreo no probabilístico por conveniencia, entre febrero y noviembre de 2009. En cada predio se examinaron todos los recipientes capaces de contener agua, tales como albercas, canecas y floreros, entre otros, recolectando en ellos todas las larvas y pupas vivas macroscópicamente relacionadas con A. aegypti. Este material se transportó dentro de las siguientes 24 a 48 horas hasta el Laboratorio de Entomología del Instituto Nacional de Salud, donde se hizo la determinación taxonómicamente mediante claves (25). El material entomológico se mantuvo en condiciones ambientales controladas: temperatura de $26 \pm 2{ }^{\circ} \mathrm{C}$, humedad relativa de $60 \pm 3 \%$, fotoperíodo de 12 horas-luz: 12 horas de oscuridad.

\section{Establecimiento de colonias}

Cada población natural correspondiente a cada comuna o sector muestreado, se dispuso en cubetas plásticas de $30 \times 30 \times 8 \mathrm{~cm}$, con una cantidad aproximada de 800 a 1.000 individuos por cada dos litros de agua declorada, los cuales se alimentaron diariamente con Rodentina ${ }^{\circledR}$ pulverizada. A partir de las formas adultas de la generación $\mathrm{F} 1$, se obtuvieron las hembras para las pruebas bioquímicas, las cuales se conservaron a $-70{ }^{\circ} \mathrm{C}$ hasta la realización de los ensayos. El material entomológico restante se llevó hasta la segunda generación filial, alimentando los machos con agua azucarada al $10 \%$ y las hembras con sangre de ratón (Mus musculus, Linnaeus 1758), siguiendo los protocolos de manejo de vertebrados del Instituto Nacional de Salud.

Para la obtención de oviposturas, se introdujo en cada jaula un frasco de $200 \mathrm{ml}$ con $40 \mathrm{ml}$ de agua sin cloro rodeado por papel filtro, previamente etiquetado con la fecha, la especie, la generación y el sitio de recolección. Una vez obtenidas las oviposturas, los papeles se almacenaron en cámara húmeda durante dos días para la maduración del embrión, y se dejaron secar para su almacenaje y conservación. Cada tira se almacenó por un máximo de siete días, evaluando el porcentaje de eclosión.

\section{Ensayos biológicos}

Para los bioensayos con temefos, se siguió la metodología de la OMS (1981). La concentración diagnóstica se preparó a partir de una concentración de grado técnico de temefos de Chem Service ${ }^{\circledR}$ (West Chester, PA, USA). El diseño consistió en cuatro réplicas y un control. Cada réplica consistió en un vaso de vidrio, donde se expusieron 25 larvas de tercer estadio tardío o cuarto estadio temprano a una solución de $99 \mathrm{ml}$ de agua sin cloro y un mililitro de la dosis diagnóstica de 1,2 mg/L, completando a un volumen de $100 \mathrm{ml}$ para una concentración final de $0,012 \mathrm{mg} / \mathrm{L}$ (27). En los vasos control se agregó un mililitro de etanol absoluto de grado reactivo (Sigma), libre de insecticida. Para cada bioensayo se controló la temperatura de las soluciones $(28 \pm 2$ $\left.{ }^{\circ} \mathrm{C}\right)$, con el objeto de evitar la muerte de los insectos por choque térmico, manteniendo condiciones controladas del laboratorio a una temperatura promedio de $28 \pm 2{ }^{\circ} \mathrm{C}$ y una humedad relativa de $40 \pm 10 \%$. Los resultados se expresaron como el porcentaje de mortalidad 24 horas después de exposición al producto. Estos criterios se basaron en protocolos de la OMS (26).

En los bioensayos con adultos se siguió el método de botellas impregnadas propuesto por los Centers for Disease Control and Prevention (CDC) de Atlanta, Estados Unidos (22). Se utilizaron insecticidas de grado estándar (Chem Service,West Chester, PA, USA), los cuales se diluyeron con etanol absoluto de grado reactivo (Sigma) para obtener las siguientes concentraciones diagnósticas establecidas previamente para esta especie en Colombia: DDT, $150 \mu \mathrm{g} / \mathrm{ml}$; lambdacialotrina, 6,25 $\mu \mathrm{g} / \mathrm{ml}$; deltametrina, $6,25 \mu \mathrm{g} / \mathrm{ml}$; ciflutrina, $25 \mu \mathrm{g} /$ $\mathrm{ml}$; permetrina, $21,5 \mu \mathrm{g} / \mathrm{ml}$; fenitrotión, $75 \mu \mathrm{g} / \mathrm{ml}$, y malatión, $100 \mu \mathrm{g} / \mathrm{ml}(27,28)$.

Se introdujeron entre 20 y 28 mosquitos hembra de la generación filial 2 de tres días de nacidas y sin alimentarse de sangre, en botellas Schott ${ }^{\circledR}$ de $250 \mathrm{ml}$, impregnadas previamente con $1 \mathrm{ml}$ de esta concentración o etanol absoluto en el caso de los controles.

Las lecturas se hicieron en los siguientes tiempos diagnósticos, en minutos: 30 para DDT, 15 para lambdacialotrina, 30 para deltametrina, 15 para ciflutrina, 15 para permetrina, 45 para fenitrotión, y 30 para malatión. $(27,28)$.

Para cada bioensayo se hicieron tres repeticiones, cada una consistió en cuatro réplicas más un control con etanol. En cada bioensayo se incluyó una 
réplica, usando la cepa de referencia Rockefeller para $A$. aegypti según cada dosis diagnóstica evaluada por molécula. Todos los bioensayos con adultos se hicieron durante el 2009, en condiciones controladas de laboratorio y a una temperatura promedio de $24 \pm 2{ }^{\circ} \mathrm{C}$ y una humedad relativa de $55 \pm 5 \%$.

\section{Pruebas bioquímicas}

Por cada población natural del mosquito que hubiese registrado pérdida de sensibilidad a, por lo menos, uno de los insecticidas evaluados mediante bioensayos, se seleccionaron treinta mosquitos hembra de tres días de emergidas y sin alimentar, con el propósito de evaluar la actividad de $\beta$-esterasas inespecíficas y enzimas P450 $(29,30)$. Las muestras se homogenizaron individualmente en solución tampón de fosfato de potasio a $\mathrm{pH}$ 7,2 . De este preparado, se sirvieron $100 \mu \mathrm{l}$ del homogenizado en cada pozo de microplaca para practicar un ELISA. Para determinar el incremento de las $\beta$-esterasas inespecíficas, se empleó un éster- $\beta$-naftil-acetato (Merck) como sustrato, en tanto que para las enzimas P450 se utilizó peróxido de hidrógeno (Microgen Chemicals).

Los tres controles positivos por cada placa de ELISA consistieron en citocromo $C$ para P450 y $\beta$-naftol para $\beta$-esterasas inespecíficas, en tanto que los tres controles negativos consistieron en solución tampón de fosfato de potasio a pH 7,2. En cada población se midieron las densidades ópticas mediante el lector de ELISA (Thermo Labsystems) con filtro de $570 \mathrm{~nm}$ para las $\beta$-esterasas inespecíficas y de $630 \mathrm{~nm}$ para las enzimas P450. La concentración de proteínas se determinó para cada muestra por medio del método Bradford (31), con el objeto de ajustar las diferencias en la masa corporal de los individuos que pudieran requerir factores de corrección $(32,33)$. Cada muestra se analizó por triplicado a temperatura ambiente.

\section{Interpretación de los datos}

Los bioensayos que registraron porcentajes de mortalidad en los controles superiores a $20 \%$, se invalidaron. Aquellos con valores de mortalidad en el control entre 5 y $20 \%$, se ajustaron usando la fórmula de Schneider-Orelli: \% mortalidad corregido $=[(\%$ de mortalidad del tratamiento $-\%$ de mortalidad del control) / (\% de 100-mortalidad del control) $\times$ 100] (34).

Para los ensayos biológicos de los CDC, se tuvo en cuenta el criterio de mortalidad propuesto por Brogdon y McAllister (35), incluyendo en la mortalidad insectos con dificultad para volar o para sostenerse en pie al momento de rotar la botella y los que caían evidentemente muertos. La disminución de la mortalidad obtenida con la concentración de cada insecticida, indicó una disminución en la sensibilidad.

En todos los casos, los valores de mortalidad inferiores a $80 \%$ se asociaron con resistencia a insecticidas; aquellos entre 80 y $90 \%$ indicaron que las poblaciones deben mantenerse en vigilancia sistemática, en tanto que, entre 90 y $100 \%$, se consideraron sensibles.

Para determinar la actividad enzimática en las poblaciones con registro de pérdida de sensibilidad, se evaluó la distribución de frecuencias del resultado de las densidades ópticas de cada población respecto a la cepa de referencia Rockefeller. Para determinar el incremento en el nivel de $\beta$-esterasas inespecíficas y enzimas $\mathrm{P} 450$, se establecieron puntos de corte usando la cepa de referencia Rockefeller de $A$. aegypti. Los promedios de las densidades ópticas de las poblaciones de campo, iguales o superiores a este valor, se consideraron como normales, en tanto que los valores superiores se asumieron como incrementos en el nivel de esterasas inespecíficas y de enzimas P450.

Se determinó estadísticamente si los datos de las poblaciones cumplían una distribución normal de acuerdo con el tamaño de la muestra, pero al no cumplirse estos criterios, se analizó la información mediante pruebas no paramétricas con las cuales se compararon las medianas de las poblaciones con la prueba de Kruskal-Wallis (36) y, posteriormente, se compararon las poblaciones de campo con la cepa de referencia Rockefeller mediante la prueba de Mann-Whitney (36). Esta información se procesó en los programas Minitab ${ }^{\circledR}$, versión 16, y Excel Microsoft Office $2010^{\circledR}$.

\section{Resultados}

\section{Ensayos biológicos}

Todas las poblaciones registraron sensibilidad a los insecticidas organofosforados, tanto con la técnica de los CDC para adultos (figura 2, cuadro 1) como con la técnica de la OMS para larvas (cuadro 2). Todas las poblaciones presentaron pérdida de sensibilidad a lambdacialotrina y a permetrina (cuadro 1). Dos de las siete poblaciones registraron sensibilidad a deltametrina y en cinco poblaciones se presentó sensibilidad a ciflutrina. Se registró pérdida de la sensibilidad al DDT en las siete poblaciones evaluadas. 

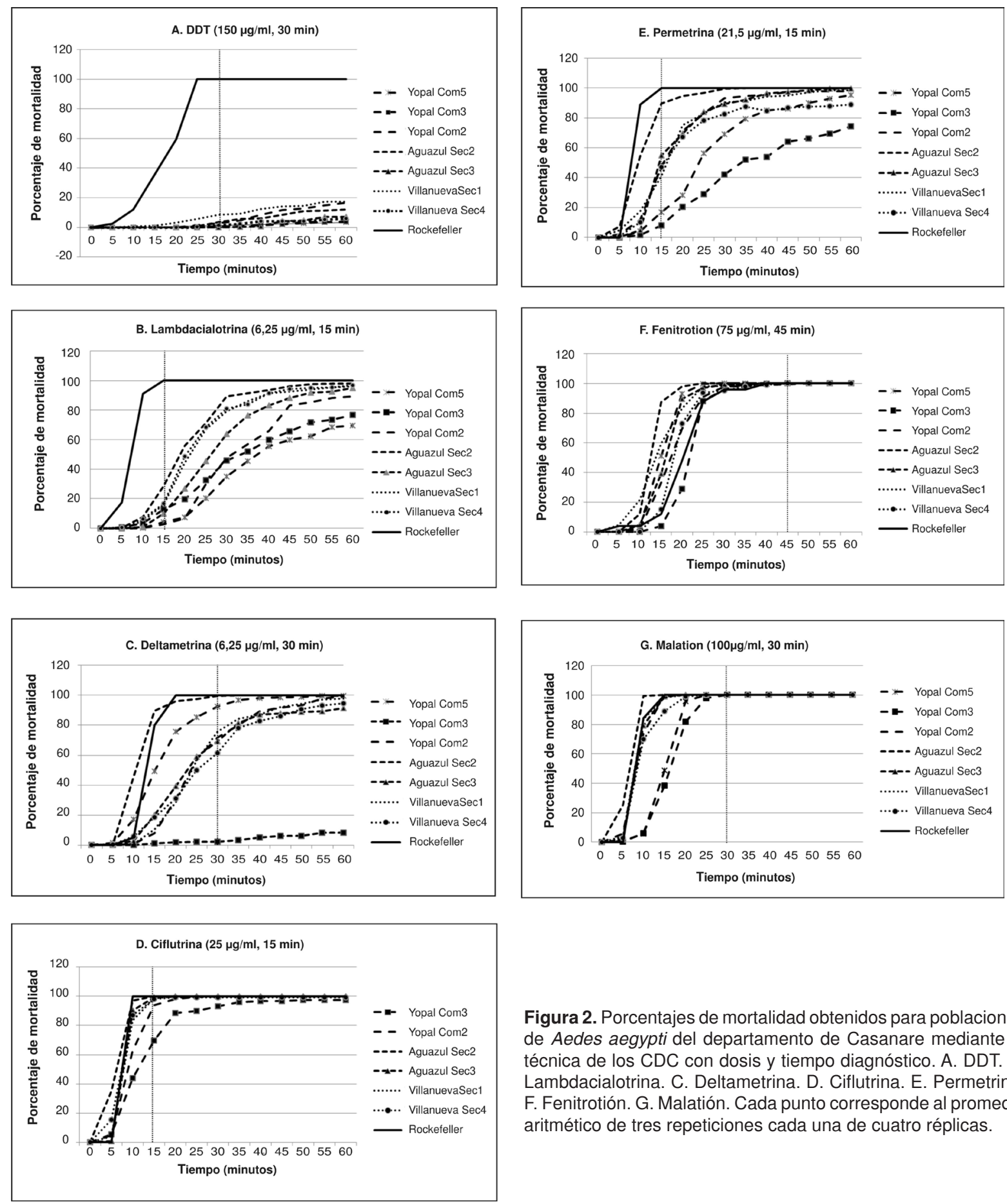

Figura 2. Porcentajes de mortalidad obtenidos para poblaciones de Aedes aegypti del departamento de Casanare mediante la técnica de los CDC con dosis y tiempo diagnóstico. A. DDT. B. Lambdacialotrina. C. Deltametrina. D. Ciflutrina. E. Permetrina. F. Fenitrotión. G. Malatión. Cada punto corresponde al promedio aritmético de tres repeticiones cada una de cuatro réplicas.

\section{Pruebas bioquímicas}

La actividad enzimática de las $\beta$-esterasas inespecíficas presentó diferencias significativas entre las medianas de las poblaciones evaluadas $(p<0,05)$. Las poblaciones que no presentaron diferencias significativas respecto a la cepa de referencia, correspondieron a Yopal Com 2

( $p=0,767)$ y Aguazul Sec $2(p=0,0774)$ (figura 3). Ninguna de las poblaciones superó el punto de corte de 0,67 , lo que se interpretó como una no alteración de las $\beta$-esterasas inespecíficas.

Aunque todas las poblaciones presentaron diferencias significativas para las $\mathrm{P} 450$ respecto a la cepa de referencia Rockefeller $(p<0,05)$, 
Cuadro 1. Promedios de los porcentajes de la mortalidad de siete poblaciones de mosquitos de Aedes aegypti a dosis y a tiempo diagnóstico para cada uno de los insecticidas evaluados, con la metodología de los CDC, Casanare, 2009

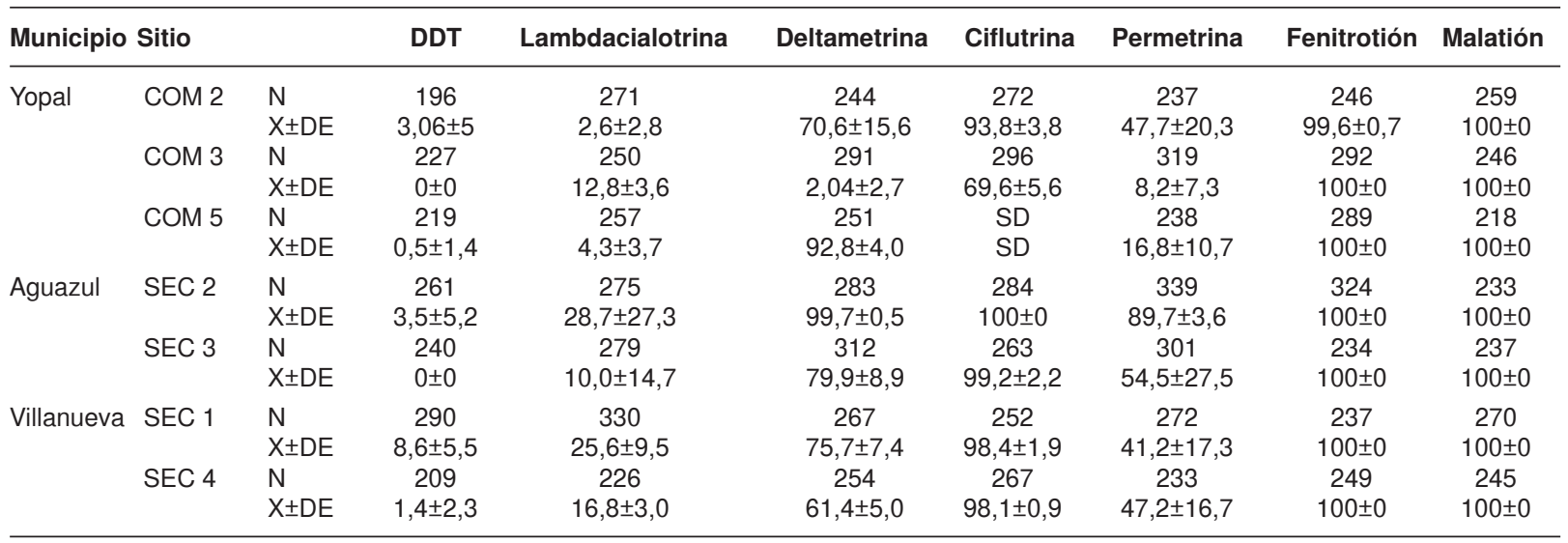

DE: desviación estándar

Cuadro 2. Promedios de los porcentajes de la mortalidad de siete poblaciones de mosquitos de $A$. aegypti Casanare, 2009 evaluados para el larvicida temefos, con la metodología de la OMS, 1981

\begin{tabular}{lccc}
\hline Municipio & Sitio & \multicolumn{2}{c}{ Temefos } \\
\hline Yopal & COM 2 & $\mathrm{N}$ & 247 \\
& & $\mathrm{X} \pm \mathrm{DE}$ & $100 \pm 0$ \\
& COM 3 & $\mathrm{N}$ & 291 \\
& & $\mathrm{X} \pm \mathrm{DE}$ & $100 \pm 0$ \\
& COM 5 & $\mathrm{N}$ & 405 \\
& & $\mathrm{X} \pm \mathrm{DE}$ & $99,5 \pm 2,3$ \\
Aguazul & SEC 2 & $\mathrm{N}$ & 305 \\
& & $\mathrm{X} \pm \mathrm{DE}$ & $100 \pm 0$ \\
& SEC 3 & $\mathrm{N}$ & 289 \\
& & $\mathrm{X} \pm \mathrm{DE}$ & $89,9 \pm 9,0$ \\
Villanueva & SEC 1 & $\mathrm{N}$ & 285 \\
& & $\mathrm{X} \pm \mathrm{DE}$ & $100 \pm 0$ \\
& SEC 4 & $\mathrm{N}$ & 301 \\
& & $\mathrm{X} \pm \mathrm{DE}$ & $93,7 \pm 6,6$ \\
\hline
\end{tabular}

solamente las poblaciones de Yopal (Com2 y Com5) superaron significativamente el valor del punto de corte de 0,323 (figura 4), lo que se interpretó como un incremento de la acción de las enzimas p450 en los mecanismos bioquímicos relacionados con la desintoxicación a las moléculas de insecticidas evaluadas.

\section{Discusión}

En el presente estudio se evaluó la sensibilidad de poblaciones naturales de $A$. aegypti a los principales insecticidas autorizados por el programa nacional de control y los más usados por la comunidad de tres municipios endémicos para dengue en el departamento del Casanare de la Orinoquia colombiana. Durante los últimos cincuenta años, los insecticidas de síntesis química han sido la estrategia de elección para el control de vectores transmisores de enfermedades. Los organofosforados han sido ampliamente usados en el país, aplicados como adulticidas a volumen ultrabajo durante picos de alta transmisión o como larvicida aplicado en los contenedores de agua potenciales criaderos del mosquito (19). Aunque en Colombia se ha registrado resistencia genética generalizada al organofosforado temefos (13-16), en este estudio se encontró que las poblaciones naturales del vector del dengue en tres municipios de Casanare se comportaron como sensibles a los organofosforados temefos, malatión y fenitrotión.

El uso de organofosforados, como el malatión y el temefos, en el departamento de Casanare se implementó a mediados de la década de 1990, y desde esa fecha ha sido el único grupo químico empleado por las entidades de salud del Estado para el control de brotes por dengue, pese al inconformismo por parte de la comunidad frente al uso del malatión a consecuencia de su olor y acción corrosiva sobre el hierro, el acero, la hojalata y el cobre, y sumado a esto, a la suspensión de este insecticida con hidrocarburos tambien corrosivos.

Por el contrario, el uso del temefos ha tenido mejor aceptación en la comunidad dado que su acción de choque contundente contra las larvas es evidenciado por la población, sin las consecuencias negativas del malatión. Estos productos se emplean solamente como medida de choque en brotes epidémicos de dengue y no como una medida regular en el departamento, por lo que se hace relevante el trabajo multisectorial y participativo mediante el control físico y biológico de criaderos potenciales del mosquito, evitando entre otras consecuencias la resistencia a este grupo químico, 


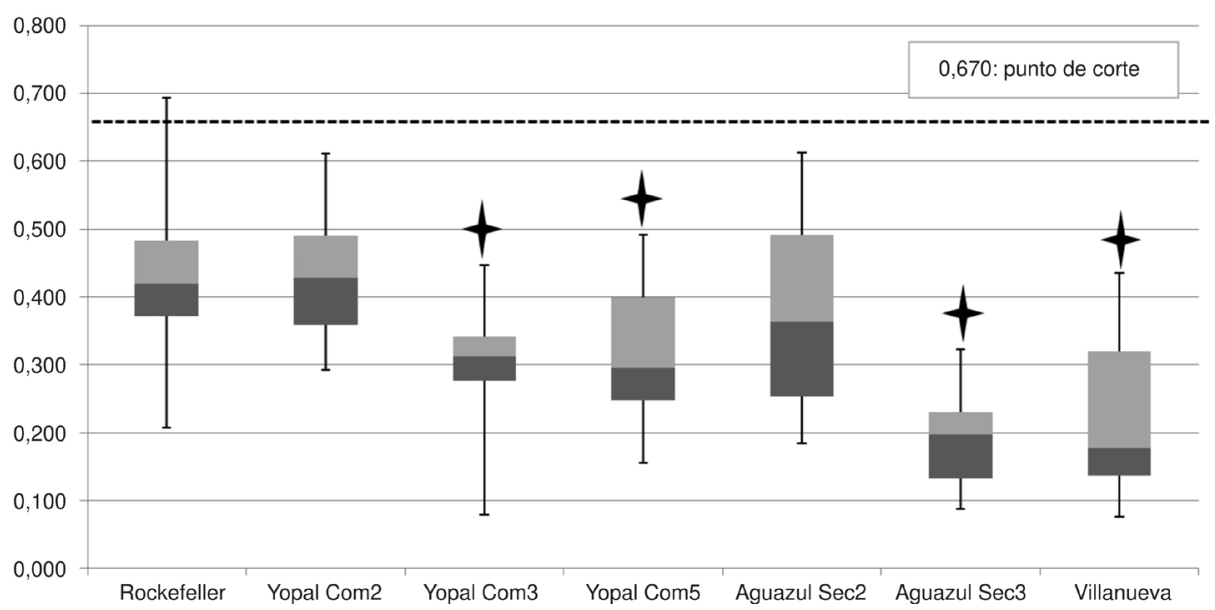

Figura 3. Diagrama de cajas de los valores de absorbancia para la actividad enzimática de las $\beta$-esterasas inespecíficas. Poblaciones con diferencia significativa respecto a la cepa Rockefeller de referencia marcadas con estrella.

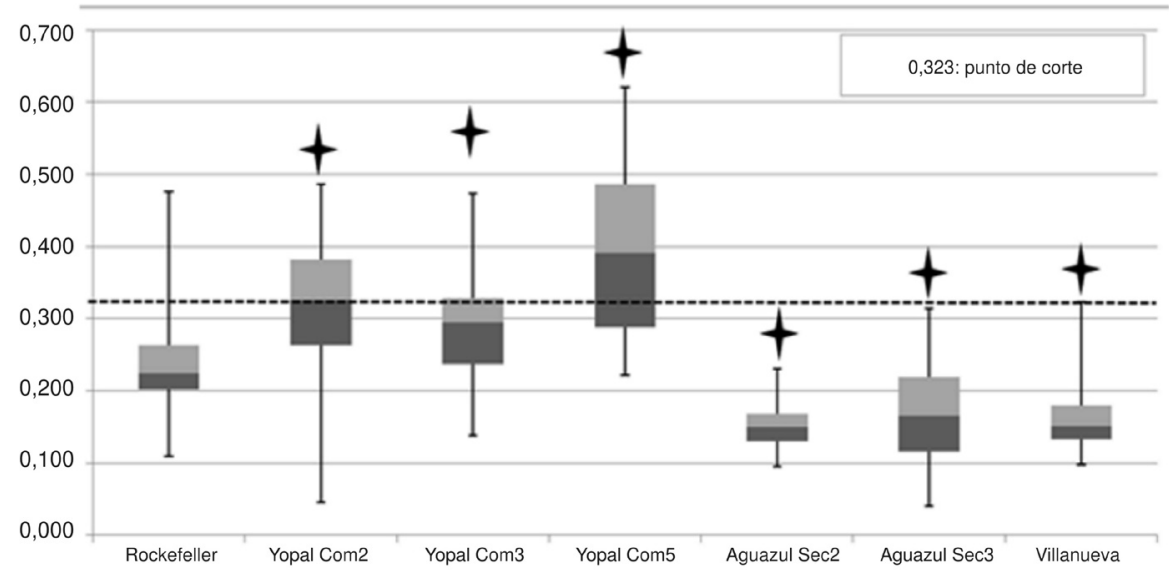

Figura 4. Diagrama de cajas de los valores de absorbancia para la actividad enzimática de las P450. Poblaciones con diferencia significativa respecto a la cepa Rockefeller de referencia marcadas con estrella.

como es el caso actual de poblaciones resistentes al temefos en Colombia (13-15), así como en Cuba (37), Brasil (38), Perú (39), Venezuela $(9,40)$ y Argentina (41).

En Colombia, la prohibición de productos clorados se inició en la década de 1970 con la restricción para los cultivos de tabaco (Resolución 447 de 1974) y café (Resolución 209 de 1978) (42), siendo finalmente prohibido únicamente en agricultura en 1986 (Resoluciones 209/78 y 447/74). Sin embargo, en 1993 Colombia fue el primer país de Latinoamérica en suspender su uso en salud pública (Resolución 10225), además de restringuir también los insecticidas dieldrín, clordano, dodecacloro, fentaclorofenol, dicofol, hexacloruro de benceno (BHC), heptacloro y linda (42). En Casanare se usó hasta comienzos de 1990 para el control de vectores de malaria y de dengue.
En el presente estudio se reveló una contundente resistencia a este insecticida en todas las poblaciones evaluadas. El continuo uso de este insecticida y otros organoclorados en el país, no solo para el control de enfermedades de interés en salud pública, sino para uso agrícola, pudo generar resistencia en poblaciones de mosquitos $A$. aegypti y fijar alelos resistentes. En general, para el vector del dengue se asume que la reinfestación de este mosquito a partir de poblaciones provenientes de Norte de Santander, donde se identificó por primera vez resistenciaaDDTen 1959(43), constituyeel principal factor para encontrar resistencia generalizada mediante mecanismos de herencia y fijación en el genoma de todas las poblaciones naturales presentes en Colombia (44). Esta resistencia es evidente en otras localidades estudiadas del país (13-16) y tambien se ha registrado en 23 naciones, 
entre ellas Trinidad, Puerto Rico, Jamaica, Haití, República Dominicana, Venezuela y Surinam $(17,43)$. La resistencia a este insecticida está mediada principalmente por mutaciones del gen $k d r$, así como con el incremento de enzimas como la glutation-S-transferasa, cuyo gen GSTe2 mostró sobreexpresión en poblaciones de Colombia (15) $y$, en menor medida, por responsabilidad del grupo citocromo P450, el cual se ha encontrado asociado principalmente a la resistencia a los piretroides (45).

El comportamiento de la mortalidad frente a los piretroides fue variable. Aunque estos no se emplean actualmente para el control de brotes epidémicos de dengue, la deltametrina y la cipermetrina se emplearon en las décadas de 1970 y 1980 en algunos municipios para el control de casos periurbanos de malaria, y se usan en la actualidad como ingrediente activo de insecticidas caseros.

En el presente estudio se encontró resistencia a la lambdacialotrina en todas las poblaciones naturales del vector del dengue de Casanare evaluadas, al igual que lo encontrado en otras localidades del país (13-16), así como en las Islas Caimán (45), Puerto Rico (46) Cuba, Perú (47) y otros países como Vietnam (48). El uso de este insecticida se intensificó en Casanare para el control de vectores de la malaria y la enfermedad de Chagas en áreas rurales, luego de la restricción del DDT. El empleo de este químico como alternativa para el control del dengue en el departamento del Casanare, eventualmente no sería recomendable a pesar de que en otros países de América han podido emplearlo con éxito, como es el caso de Costa Rica, donde se logró una letalidad de 97 a $100 \%$, tanto en tratamientos de volumen ultrabajo como en tratamientos térmicos (49).

Por otro lado, también se evidenció resistencia generalizada a la permetrina. Si bien este insecticida no se emplea por los programas de salud pública para el control del vector del dengue en Colombia, esta molécula constituye el ingrediente activo de uno de los insecticidas caseros más comunes de venta libre en el país, el cual es de uso frecuente en las localidades evaluadas del departamento de Casanare. En poblaciones de Chocó y Putumayo, la pérdida de sensibilidad del vector del dengue se asoció al uso de esta molécula para el control de vectores de la malaria a partir de su incorporación en toldillos de larga duración (15), situación diferente a la presentada en Casanare, ya que los toldillos allí empleados se impregnan con deltametrina con el objeto de proteger a la población rural de otros eventos como enfermedad de Chagas y malaria. En el Meta, un departamento próximo a Casanare y con un intercambio económico y social importante, se presentó resistencia a lambdacialotrina al igual que al DDT, lo que eventualmente podría sugerir que las poblaciones de mosquitos comparten un mecanismo de resistencia similar que les confiere protección contra estos dos químicos. Esta resistencia a los piretroides constituye una amenaza grave a las alternativas de control del mosquito, por lo que se siguen investigando mecanismos responsables, entre los cuales se ha concluido recientemente que la sobreexpresión del gen P4509J32 de P450 se ha relacionado con la resistencia a los piretroides, el cual metaboliza tanto permetrina como deltametrina en $A$. aegypti (5052). Aun así, esta resistencia tambien podría estar mediada de otras formas, como por la glutatiónS-transferasa, la $\beta$-esterasas inespecíficas y la mutación del gen $k d r(52)$.

Actualmente, en Casanare el uso de la deltametrina está vinculado al control de la enfermedad de Chagas y eventuales casos de malaria, solamente en las áreas rurales; años atrás, hasta mediados de la década de 1990, se empleó para el control de mosquitos en áreas urbanas. En este estudio, se encontró resistencia en cinco de las siete poblaciones evaluadas y una situación similar se evidenció en otras localidades de Colombia $(13,14)$. En el departamento del Meta, se reportó resistencia a este compuesto en una sola población, mientras que en Casanare fue más generalizada. Al parecer, la resistencia a deltametrina se ha asociado con el incremento de glutatión-S-transferasa, de $\beta$-esterasas inespecíficas, de enzima P450 y por mutaciones puntuales del gen $k d r$, pudiéndose presentar resistencia cruzada por algún tipo de mecanismo en algunas de las poblaciones (53). Sin embargo, es importante resaltar que la presión en las poblaciones de mosquitos por organofosforados, puede generar a largo plazo resistencia a otros grupos piretroides, como la deltametrina $(47,53)$.

A la ciflutrina, por su parte, se presentó resistencia fisiológica solo en dos de las poblaciones del municipio de Yopal. Este dato en Colombia solo es comparable con los resultados arrojados por Fonseca, et al., quienes encontraron sensibilidad en todas las poblaciones evaluadas y en otras localidades del Perú y de Cuba (47). Este químico está aprobado por la OMS para el uso en salud pública y puede ser usado en aerosol en frío 
y niebla caliente para el control de mosquitos adultos (20); además, actualmente es componente activo de uno de los insecticidas caseros más frecuentemente usados en los municipios evaluados y, probablemente, debido a su reciente uso en el mercado respecto a los demás químicos, pudo presentar mayor sensibilidad entre los piretroides. El uso comercial de este producto es regulado mediante la resolución No. 1386 del 16 de julio de 2010 del Ministerio de Ambiente, Vivienda y Desarrollo Territorial.

La actividad enzimática de las $\beta$-esterasas inespecíficas en todas las siete poblaciones evaluadas, se encontró por debajo del punto de corte establecido y por debajo del promedio de la cepa de referencia Rockefeller, por lo cual se sugiere que no se presenta una asociación de esta enzima con la resistencia a los grupos de los organofosforados ni piretroides. Este resultado es contrario a los de estudios previos en el país (13-15) y otras localidades de América $(46,47)$, donde precisamente se encontró incremento en la actividad de esta familia de enzimas, lo que sugiere que podría estar asociado con la resistencia al temefos así como otros piretroides como lambdacialotrina $(8,13,53-$ 55). Es probable, entonces, que se presenten otros mecanismos de resistencia diferentes a los evaluados, como la actividad de la glutatión-Stransferasa que pudieran, no solo estar confiriendo resistencia al DDT, sino también protección a las células de los mosquitos contra los efectos tóxicos de los piretroides, mediante actividad peroxidasa 0 por mutaciones puntuales del gen $k d r$, como en el caso de otros piretroides.

Se evidenció un incremento significativo en la actividad de enzimas P450 solamente en las tres comunas del municipio de Yopal, por lo que se sugiere una posible asociación con la pérdida de sensibilidad a los piretroides evaluados. En Colombia, este incremento se evidenció igualmente en otras localidades (13-15) y se asoció con la resistencia a organofosforados. Estas enzimas P450 son una línea vital de defensa de los insectos contra los miles de químicos de origen natural que tienen que evadir, por lo que puede mediar la resistencia a piretroides y carbamatos, y en menor medida, a organofosforados y organoclorados.

No obstante, esto no explicaría del todo la pérdida de sensibilidad encontrada en las restantes poblaciones evaluadas, por lo que se sugiere la presencia de otros mecanismos de resistencia involucrados que también puedan estar actuando en ellas. El mecanismo de resistencia de P450 es muy complejo; hasta la fecha se han detectado 160 genes de desintoxicación en el genoma de este mosquito (56). Aun así, es probable que este mecanismo no sea la principal causa de esta resistencia; para aclararlo, es indispensable ampliar este estudio con pruebas sinergistas y pruebas moleculares, y poder determinar la posibilidad de otros mecanismos involucrados, como las mutaciones en el canal de sodio encontradas en poblaciones de las Islas Caimán, donde a pesar de encontrarse un incremento en la actividad de glutatión-S-transferasa, $\beta$-esterasas inespecíficas y $\mathrm{P} 450$, la causa de esta resistencia se debió a mutaciones por sustitución en el sitio blanco (46), por lo que se hace importante adelantar estudios moleculares para determinar el papel del gen $k d r$ en la resistencia a piretroides y DDT.

Este aumento de la actividad enzimática puede producirse por amplificación de genes, sobreexpresión, mutaciones de secuencias o por una combinación de estos mecanismos. Es así como la glutatión-S-transferasa puede mediar la resistencia a organofosforados, organoclorados y piretroides, la familia de esterasas pueden proporcionar resistencia a organofosforados, carbamatos y piretroides; esta gran diversidad genética ha podido causar una amplia especificidad en el sustrato en las enzimas metabólicas de los insectos. Y, así, se observa que la pérdida de la sensibilidad a diferentes moléculas es un fenómeno que varía a nivel local, entre poblaciones, y puede depender tanto de los componentes genéticos, como de la historia de presión de selección ejercida por el uso de insecticidas.

De esta manera, se sugiere que el uso de organofosforados en el departamento de Casanare se debe continuar de una manera racional y focalizada de acuerdo con lineamientos nacionales, ya que las alternativas para la rotación de productos no es amplia y se limitaría al uso de la ciflutrina y, en algunos casos, la deltametrina. Es relevante, igualmente, fortalecer los programas de Información Comunicación y Educación, con el fin de que la misma comunidad tenga una participación activa en los programas de control implementados y fortalecer así las actividadades de control físico para disminuir las poblaciones de este mosquito en el departamento. Aunque se presente sensibilidad en las poblaciones de mosquitos, es importante vigilarlas de manera regular y ampliar su selección en localidades con transmisión de la enfermedad. 
Este trabajo contribuye a consolidar este departamento dentro de la Red Nacional de Vigiancia de la Resistencia a los insecticidas usados para el control de vectores en el país y se espera que estos resultados sirvan como punto de partida para futuros estudios sobre resistencia en el departamento, así como para fortalecer el componente educativo en la comunidad enfocando los esfuerzos al control físico de los criaderos potenciales de cada municipio.

\section{Agradecimientos}

Los autores expresan los agradecimientos a la Secretaría de Salud de Casanare y al Instituto Nacional de Salud, por el apoyo institucional y la colaboración del personal técnico y profesional en la realización de este trabajo.

\section{Conflicto de intereses}

Los autores declaramos que no existen conflictos de intereses en la realización y difusión de este trabajo.

\section{Financiación}

Este trabajo fue financiado por la Secretaría de Salud de Casanare, el Instituto Nacional de Salud y la Universidad Nacional de Colombia a través de la Dirección de Investigación sede Bogotá.

\section{Referencias}

1. Kroeger A, Nathan MB. Dengue: Setting the global research agenda. Lancet. 2006;368:2193-2195. http:// dx.doi.org/10.1016/S0140-6736(06)69873-5

2. Guzmán MG, Halstead SB, Artsob H, Buchy P, Farrar J, Gubler DJ, et al. Dengue: A continuing global threat. Nat Rev Microbiol. 2010;8(Suppl.):S7-16. http://dx.doi. org/10.1038/nrmicro2460.

3. Instituto NacionaldeSalud.BoletínepidemiológicoSemana 52. Fecha de consulta: 12 de abril de 2013. Disponible en: http://ins.gov.co/lineas-de-accion/Subdireccion-Vigilancia/ sivigila/Paginas/vigilancia-rutinaria.aspx.

4. Instituto Nacional de Salud. Informe de enfermedades transmitidas por vectores (ETV), 2004. Inf Quinc Epidemiol Nac. 2005;10:3.

5. Instituto Nacional de Salud. Boletín epidemiológico Semana 28. Fecha de consulta: 14 de agosto de 2012. Disponible en: http://www.ins.gov.co/boletin-epidemiologico/ Boletn $\% 20$ Epidemiolgico/2012\%20Boletin $\% 20$ epidemiologico_Semana\%2028.pdf.

6. World Health Organization. Dengue fever and dengue hemorrhagic fever prevention and control. Geneva: WHO; 2002.

7. Georghiou GP, Wirth M, Tran H, Saume F, Knudsen AB. Potential for organophosphate resistance in Aedes aegypti (Diptera: Culicidae) in the Caribbean area and neighboring countries. J Med Entomol. 1987;24:290-4.
8. Mekuria Y, Gwinn TA, Williams DC, Tidwell MA. Insecticide susceptibility of Aedes aegyptifrom Santo Domingo, Dominican Republic. J Am Mosq Control Assoc. 1991;7:69-72.

9. Mazzarri MB, Georghiou GP. Characterization of resistance to organophosphate, carbamate, and pyrethroid insecticides in field populations of Aedes aegypti from Venezuela. J Am Mosq Control Assoc. 1995;11:315-22.

10. Rawlins SC, Wan JO. Resistance in some Caribbean populations of Aedes aegypti to several insecticides. J Am Mosq Control Assoc. 1995;11:59-65.

11. Brown AW. Insecticide resistance in mosquitoes: A pragmatic review. J Am Mosq Control Assoc. 1986;2:12340.

12. Michigan State University. Arthropod Pesticide Resistance Database. Fecha de consulta: 7 de septiembre de 2012. Disponible en: http://www.pesticideresistance.org/display. php?page $=$ species $\&$ arld $=7$

13. Santacoloma L, Chaves B, Brochero HL. Susceptibilidad de Aedes aegypti a DDT, deltametrina y lambdacialotrina en Colombia. Rev Panam Salud Publica. 2010;27:66-73. http:// dx.doi.org/10.1590/S1020-49892010000100010

14. Ocampo CB, Salazar-Terreros MJ, Mina NJ, McAllister J, Brogdon W. Insecticide resistance status of Aedes aegypti in 10 localities in Colombia. Acta Tropica. 2011;118:37-44. http://dx.doi.org/10.1016/j.actatropica.2011.01.007

15. Fonseca-González I, Quiñones ML, Lenhart A, Brogdon WG. Insecticide resistance status of Aedes aegypti (L.) from Colombia. Pest Manag Sci. 2011;67:430-7. http://dx.doi. org/10.1002/ps.2081

16. Maestre R, Rey G, de las Salas J, Vergara C, Santacoloma L, Goenaga S, et al. Estado de la susceptibilidad de Aedes aegypti a insecticidas en Atlántico (Colombia). Rev. Colomb. Entomol. 2010;36:242-8.

17. Brown A. The insecticide-resistance problem. A review of developments in 1956 and 1957. Bull World Health Organ. 1958;18:309-21.

18. Manotas LS, Gallego JI, Vélez ID. Especies del género Lutzomyia (Psychodidae: phlebotominae): distribución espacial y densidad relativa, Casanare - Colombia. Portales médicos. 2012;7. Fecha de consulta: 4 de noviembre del 2012. Disponible en: http://www.portalesmedicos. com/publicaciones/articles/4140/1/Especies-del-generoLutzomyia---psychodidae-phlebotominae-Distribucionespacial-y-densidad-relativa.html.

19. Ministerio de la Protección Social, Instituto Seccional de Salud de Boyacá. Guía integral de manejo de las enfermedades transmitidas por vectores. Tunja: Editorial Muisca; 2003. p. 114.

20. Organización Mundial de la Salud. Dengue: guías para el diagnóstico, tratamiento, prevención y control. La Paz: OMS; 2009. p. 152.

21. Gobernación de Casanare. Localización de Casanare. Fecha de consulta: 21 de agosto de 2012. Disponible en: http://www.casanare.gov.co/?idcategoria=1196.

22. Gobernación de Casanare, CORPORINOQUIA, UAESPNN Y CORPOBOYACÁ. Plan de ordenación y manejo de la cuenca del río Cravo Sur. Yopal: Gobernación de Casanare; 2007. p. 969. 
23. Nelson MJ. Aedes aegypti: biología y ecología. Washington, D.C: Organización Panamericana de la Salud; 1986; 50.

24. Martínez FC. Los mosquitos de México (Diptera: Culicidae). Taxonomía, distribución geográfica y su importancia en Salud Pública (tesis). México: UNAM Facultad de Ciencias; 1987.

25. Tinker M. Clave práctica para larvas de mosquitos neotropicales en recipientes. Bogotá: DANIDA/OPS/OMS; 1983.

26. World Health Organization. Instructions for determining the susceptibility or resistance of mosquitoes larvae to insecticide. Geneva: WHO; 1981.

27. Fonseca I. Estatus de la resistencia a insecticidas de los vectores primarios de malaria y dengue en Antioquia, Chocó, Norte de Santander y Putumayo, Colombia (tesis). Medellín: Universidad de Antioquia; 2008.

28. Santacoloma L. Estado de la susceptibilidad a insecticidas de poblaciones naturales de Aedes aegypti Linnaeus, 1762 vector del dengue y Anopheles darlingi Root, 1926 vector primario de malaria (Diptera: Culicidae) en cinco departamentos de Colombia (tesis). Bogotá: Universidad Nacional de Colombia; 2008. ¿Páginas?

29. Brogdon WG, Barber AM. Fenitrothion-deltamethrin crossresistance conferred by esterases in Guatemalan Anopheles albimanus. Pest Biochem Physiol. 1990;37:130-9. http:// dx.doi.org/10.1016/0048-3575(90)90118-L

30. Brogdon WG, McAllister JC and Vulule J. Heme peroxidase activity measured in single mosquitoes identifies individuals expressing an elevated oxidase for insecticide resistance. J Am Mosq Control Assoc. 1997;13:233-7.

31. Bradford M. A rapid and sensitive method for the quantitation of microgram quantities of protein dye binding. Anal Biochem. 1976;72:248-54. http://dx.doi.org/10.1016/00032697(76)90527-3

32. Brogdon WG. Mosquito protein microassay I. Protein determinations from small portions of single-mosquito homogenates. Comp Biochem Physiol A. 1984;79:457-9. http://dx.doi.org/10.1016/0305-0491(84)90405-X

33. Brogdon WG. Mosquito protein microassay II. Modificationfor potential field use. Comp Biochem Physiol B. 1984;79:461-4 http://dx.doi.org/10.1016/0305-0491(84)90406-1

34. Püntener, W. Manual for field trials in plant protection. Second edition. Switzerland: Ciba Geigy Limited Basel 1981. p. 205

35. Brogdon W, Mcallister J. Simplification of adult mosquito bioassays through use of time-mortality determinations in glass bottles. J Am Mosq Control Assoc. 1998;14:15964.

36. Moore DE. Estadística básica aplicada. Barcelona: Antony Bosch Editor; 2000. p. 836.

37. Rodríguez MM, Bisset JA, Mila L, Calvo E, Díaz C, Soca LA. Niveles de resistencia a insecticidas y sus mecanismos en una cepa de Aedes aegypti de Santiago de Cuba. Rev Cubana Med Trop. 1999;51:83-8.

38. Braga IA, Pereira JB, Da Silva S, Valle D. Aedes aegypt resistance to Temephos during 2001 in several municipalities in the state of Rio de Janeiro, Sergipe, and Halagaos, Brazil. Mem Inst Oswaldo Cruz. 2004;99:199-203. http://dx.doi. org/10.1590/S0074-02762004000200015
39. Chávez J, Córdova O, Vargas F. Niveles de susceptibilidad a temefos en el vector transmisor del dengue en Trujillo, Perú. Anales de la Facultad de Medicina Lima. 2005;66:53-6.

40. Álvarez L, Briceño A, Oviedo, M. Resistencia al Temephos en poblaciones de Aedes aegypti (Diptera: Culicidae) del occidente de Venezuela. Revista Colombiana de Entomología. 2006;32:172-5.

41. Seccacini E, Lucia A, Zerba E, Licastro S, Masuh H. Aedes aegypti resistance to temephos in Argentina J. Am Mosq Control Assoc. 2008;24:608-9.

42. Instituto Colombiano Agropecuario. Subgerencia protección y regulación agrícola restricciones, prohibiciones y suspensión de registros de plaguicidas de uso agrícola en colombia. Fecha de consulta: 19 de septiembre de 2012. Disponible en: http://www.ica.gov.co/getdoc/b2e5ff99-bd8045e8-aa7a-e55f0b5b42dc/PLAGUICIDAS-PROHIBIDOS. aspx.

43. World Health Organization. Consejo directivo, XI reunión. Tema 33: Estado de la erradicacion del Aedes aegypti en las américas. Washington: WHO; 1959.

44. Fonseca I, Quiñones M, Mcallister J, Brogdon W. Mixedfunction oxidases and esterases associated with crossresistance between DDT and lambdacyhalothrin Anopheles darlingi Root, 1926 populations from Colombia. Mem Inst Oswaldo Cruz. 2009;104:18-26. http://dx.doi.org/10.1590/ S0074-02762009000100003

45. Harris AF, Rajatileka S, Ranson H. Pyrethroid resistance in Aedes aegypti from Grand Cayman. Am J Trop Med Hyg. 2010;83:277-84.http://dx.doi.org/10.4269/ajtmh.2010.090623

46. Hemmingway RG, Boddinton RG, Harris J. Mechanisms of insecticide resistance in Aedes aegypti (L.) (Diptera: Culicidae) from Puerto Rico. Bull Ent Res. 1989;79:123-30. http://dx.doi.org/10.1017/S0007485300018630

47. Rodríguez MM, Bisset JA, Fernández D. Levels of insecticide resistance and resistance mechanisms in Aedes aegypti from some Latin American countries. J Am Mosq Control Assoc. 2007;23:420-9.

48. Huong VD, Ngoc NTB. Susceptibility of Aedes aegypti to insecticides in South Vietnam. Dengue Bull. 1999;23:256-63.

49. Perich MJ, Rocha NO, Castro AL, Alfaro AW, Platt KB, Solano $\mathrm{T}$, et al. Evaluation of the efficacy of lambdacyhalothrin applied by three spray application methods for emergency control of Aedes aegypti in Costa Rica. J Am Mosq Control Assoc. 2003;19:58-62.

50. Flores AE, Reyes GI, Fernández I, Sánchez FJ, García GP. Resistance to permethrin in Aedes egypti (L.) in Northern Mexico. Southwestern Entomologist. 2009;34:167-77. http:// dx.doi.org/10.3958/059.034.0207

51. Stevenson BJ, Pignatelli P, Nikou D, Paine MJ. Pinpointing P450s associated with pyrethroid metabolism in the dengue vector, Aedes aegypti: Developing new tools to combat insecticide resistance. PLoS Negl Trop Dis. 2012;6:e1595. http://dx.doi.org/10.1371/journal.pntd.0001595

52. Aponte A. Resistencia a insecticidas: mecanismos metabólicos y mutaciones puntuales del sitio blanco de los piretroides, en poblaciones de Aedes aegypti del estado de Guerrero (tesis). Tapachula: Instituto Nacional de Salud Pública; 2011. 
53. Rodríguez MM, Bisset JA, Armas Y, Ramos F. Pyrethroid insecticide-resistant strain of Aedes aegypti from Cuba induced by deltamethrin seleccion. J Am Mosq Control Assoc. 2005;21:437-45.

54. Saavedra K, Urdaneta L, Rajatileka S, Moulton M, Flores AE, Fernández I, et al. A mutation in the voltagegated sodium channel gene associated with pyrethroid resistance in Latin American Aedes aegypti, Insect Mol Biol. 2007;16:785-98. http://dx.doi.org/10.1111/j.13652583.2007.00774.x
55. Lima E, Santos MH, Araújo A, Gomes EV, Da Silva UM, Oliveira LN, et al. Insecticide resistance in Aedes aegypti populations from Ceará, Brazil. Parasit Vectors. 2011;4:5. http://dx.doi.org/10.1186/1756-3305-4-5.

56. Strode C, Wondji CS, David JP, Hawkes NJ, Lumjuan $\mathbf{N}$, Nelson DR, et al. Genomic analysis of detoxification genes in the mosquito Aedes aegypti. Insect Biochem Mol Biol. 2008;38:113-23. http://dx.doi.org/10.1016/j. ibmb.2007.09.007, 